Jolanta Borowska, Lena Łacińska, Jowita Rychlewska, Application of difference equations to certain tridiagonal matrices, Scientific Research of the Institute of Mathematics and Computer Science, 2012, Volume 11, Issue 3, pages 15-20.

The website: http://www.amcm.pcz.pl/

Scientific Research of the Institute of Mathematics and Computer Science 3(11) 2012, 15-20

\title{
APPLICATION OF DIFFERENCE EQUATIONS TO CERTAIN TRIDIAGONAL MATRICES
}

\author{
Jolanta Borowska, Lena Lacińska, Jowita Rychlewska \\ Institute of Mathematics, Czestochowa University of Technology \\ Czestochowa, Poland \\ jolanta.borowska@im.pcz.pl,lena.lacinska@im.pcz.pl,jowita.rychlewska@im.pcz.pl
}

\begin{abstract}
In this paper we present an application of second order homogeneous linear difference equations with constant coefficients to evaluate the determinant of tridiagonal matrices. Comparing the obtained results with a certain alternative approach [1] some formulae for the finite sum are derived.
\end{abstract}

\section{Introduction}

It is well known that the determinants of tridiagonal matrices play an important role in many applications, for example, in parallel computing or in finite differences method [2]. Moreover, there are many connections between determinants of tridiagonal matrices and the Fibonacci and Lucas numbers [3,4]. Under certain conditions the recurrence relation for computing the determinant of tridiagonal matrices was obtained in [5]. form

In paper [1] the authors considered the determinant of tridiagonal matrix of the

$$
\mathbf{A}_{n}=\left[\begin{array}{ccccccc}
a & b & 0 & 0 & \ldots & 0 & 0 \\
b & a & b & 0 & \ldots & 0 & 0 \\
0 & b & a & b & \ldots & 0 & 0 \\
0 & 0 & b & a & \ldots & 0 & 0 \\
\vdots & \vdots & \vdots & \vdots & \ddots & \vdots & \vdots \\
0 & 0 & 0 & 0 & \ldots & a & b \\
0 & 0 & 0 & 0 & \ldots & b & a
\end{array}\right]_{n \times n}
$$

It was shown that the determinant of matrix $\mathbf{A}_{n}$ is given by the formula

$$
\operatorname{det} \mathbf{A}_{n}=a^{n}-\left(\begin{array}{c}
n-1 \\
1
\end{array}\right) a^{n-2} b^{2}+\left(\begin{array}{c}
n-2 \\
2
\end{array}\right) a^{n-4} b^{4}-\left(\begin{array}{c}
n-3 \\
3
\end{array}\right) a^{n-6} b^{6}+\ldots
$$


Considerations of this paper are concerned with the determinant of the form

$$
W_{n}=\left|\begin{array}{ccccccc}
a_{2} & a_{3} & 0 & 0 & \ldots & 0 & 0 \\
a_{1} & a_{2} & a_{3} & 0 & \ldots & 0 & 0 \\
0 & a_{1} & a_{2} & a_{3} & \ldots & 0 & 0 \\
0 & 0 & a_{1} & a_{2} & \ldots & 0 & 0 \\
\vdots & \vdots & \vdots & \vdots & \ddots & \vdots & \vdots \\
0 & 0 & 0 & 0 & \ldots & a_{2} & a_{3} \\
0 & 0 & 0 & 0 & \ldots & a_{1} & a_{2}
\end{array}\right|_{n \times n}
$$

where $a_{1}, a_{2}, a_{3} \in \mathbb{C}$.

It will be shown that the problem of calculation of the determinant (3) leads to solving a second order homogeneous linear difference equation with constant coefficients.

Moreover, for special cases, the obtained results will be compared with those of [1] in order to derive formulae for certain finite sums.

\section{The main results}

In this section we are going to give the results concerning the determinant of tridiagonal matrices of the form (3). Let us observe that

$$
\begin{aligned}
& W_{1}=a_{2} \\
& W_{2}=a_{2}^{2}-a_{1} a_{3}
\end{aligned}
$$

Using the method of Laplace expansion with respect to the first column and subsequently with respect to the first row we obtain

$$
W_{n}=a_{2} W_{n-1}-a_{1} a_{3} W_{n-2}, n>2
$$

The above expression can be rewritten in the following form

$$
W_{n+2}-a_{2} W_{n+1}+a_{1} a_{3} W_{n}=0, n>0
$$

Let us observe that equation (6) is a second-order homogeneous linear difference equation with constant coefficients together with initial conditions of form (4). Following [6] we have that the general solution of the equation (6) is determined by the roots of the quadratic equation

$$
\lambda^{2}-a_{2} \lambda+a_{1} a_{3}=0, \lambda \in \mathbb{C}
$$

which depend on the discriminant 


$$
\Delta=a_{2}^{2}-4 a_{1} a_{3}
$$

Case 1. Let $\Delta \neq 0$. Then quadratic equation (7) has two different complex roots

$$
\lambda_{1}=\frac{a_{2}-\sqrt{\Delta}}{2}, \lambda_{2}=\frac{a_{2}+\sqrt{\Delta}}{2}
$$

In this case the general solution of the equation (6) has the form

$$
W_{n}=C_{1} \lambda_{1}^{n}+C_{2} \lambda_{2}^{n}
$$

Bearing in mind the initial conditions (4) we obtain the system of linear equations

$$
\left\{\begin{array}{l}
C_{1} \lambda_{1}+C_{2} \lambda_{2}=a_{2} \\
C_{1} \lambda_{1}^{2}+C_{2} \lambda_{2}^{2}=a_{2}^{2}-a_{1} a_{3}
\end{array}\right.
$$

From the above system of equations we have

$$
C_{1}=-\frac{1}{\sqrt{\Delta}} \lambda_{1} \text { and } C_{2}=\frac{1}{\sqrt{\Delta}} \lambda_{2}
$$

Substituting (9) and (12) to (10) we find that the particular solution of the equation (6) with the initial conditions (4) has the form

$$
W_{n}=\frac{1}{\sqrt{a_{2}^{2}-4 a_{1} a_{3}}}\left[\left(\frac{a_{2}+\sqrt{a_{2}^{2}-4 a_{1} a_{3}}}{2}\right)^{n+1}-\left(\frac{a_{2}-\sqrt{a_{2}^{2}-4 a_{1} a_{3}}}{2}\right)^{n+1}\right]
$$

Case 2. Let $\Delta=0$. Then the quadratic equation (7) has one double root

$$
\lambda_{1}=\lambda_{2}=\frac{a_{2}}{2}
$$

In case 2 the general solution of the equation (6) has the form

$$
W_{n}=C_{1} \lambda_{1}^{n}+C_{2} n \lambda_{1}^{n}
$$

From the initial conditions (4) we have the following system of equations

$$
\left\{\begin{array}{l}
C_{1} \lambda_{1}+C_{2} \lambda_{1}=a_{2} \\
C_{1} \lambda_{1}^{2}+C_{2} 2 \lambda_{1}^{2}=a_{2}^{2}-a_{1} a_{3}
\end{array}\right.
$$

The solution of the above system of equations has the form 


$$
C_{1}=\frac{a_{1} a_{3}}{\lambda_{1}^{2}} \text { and } C_{2}=\frac{a_{2}^{2}-2 a_{1} a_{3}}{2 \lambda_{1}^{2}}
$$

Taking into account (14) and (17) we obtain from (15) that the particular solution of the equation (6) with initial conditions (4) is given by the formula

$$
W_{n}=\frac{2 a_{1} a_{3}+n\left(a_{2}^{2}-2 a_{1} a_{3}\right)}{2}\left(\frac{a_{2}}{2}\right)^{n-2}
$$

In the subsequent analysis considerations will be restricted to the determinant in which $a_{1}=a_{3}$.

\section{Identities obtained in special cases}

The aim of this section is to derive formulae for the finite sum of certain sequences. To this end let $a_{1}=a_{3}=b, a_{2}=a, a, b \in \mathbb{C} \backslash\{0\}$. Taking into account the results obtained in paper [1] we can write the determinant of matrix (1) in the form

$$
\left.W_{n}=\sum_{k=0}^{\left\lfloor\frac{n}{2}\right\rfloor}(-1)^{k} a^{n-2 k} b^{2 k}\left(\begin{array}{c}
n-k \\
k
\end{array}\right)=a^{n} \sum_{k=0}^{\left\lfloor\frac{n}{2}\right.}\right\rfloor\left(-\frac{b^{2}}{a^{2}}\right)^{k}\left(\begin{array}{c}
n-k \\
k
\end{array}\right)
$$

where the symbol $\lfloor x\rfloor$ denotes the largest integer not greater than $x$.

Setting $c=-\frac{b^{2}}{a^{2}}, c \in \mathbb{C} \backslash\{0\}$ into formula (19), we have

$$
W_{n}=a^{n} \sum_{k=0}^{\left\lfloor\frac{n}{2}\right\rfloor} c^{k}\left(\begin{array}{c}
n-k \\
k
\end{array}\right)
$$

On the other hand, from (8) we have $\Delta=a^{2}(1+4 c)$.

It can be easily observed that if $c \in \mathbb{C} \backslash\left\{0,-\frac{1}{4}\right\}$ then $\Delta \neq 0$. In this case the formula (13) yields

$$
W_{n}=\frac{a^{n}}{\sqrt{1+4 c}}\left[\left(\frac{1+\sqrt{1+4 c}}{2}\right)^{n+1}-\left(\frac{1-\sqrt{1+4 c}}{2}\right)^{n+1}\right]
$$

Comparing (20) and (21) we have 
$\sum_{k=0}^{\left\lfloor\frac{n}{2}\right\rfloor} c^{k}\left(\begin{array}{c}n-k \\ k\end{array}\right)=\frac{1}{\sqrt{1+4 c}}\left[\left(\frac{1+\sqrt{1+4 c}}{2}\right)^{n+1}-\left(\frac{1-\sqrt{1+4 c}}{2}\right)^{n+1}\right], c \in \mathbb{C} \backslash\left\{0,-\frac{1}{4}\right\}$

If $c=-\frac{1}{4}$ then $\Delta=0$ and from (18) we have

$$
W_{n}=\frac{n+1}{2^{n}} a^{n}
$$

From the comparison of (20) and (23) we derive the following formula

$$
\sum_{k=0}^{\left\lfloor\frac{n}{2}\right\rfloor}\left(-\frac{1}{4}\right)^{k}\left(\begin{array}{c}
n-k \\
k
\end{array}\right)=\frac{n+1}{2^{n}}
$$

\section{Remark 1}

If $n$ is even, $n=2 m$, then $\left\lfloor\frac{n}{2}\right\rfloor=\left\lfloor\frac{2 m}{2}\right\rfloor=m$ and the formula (22) is given by

$$
\sum_{k=0}^{m} c^{k}\left(\begin{array}{c}
2 m-k \\
k
\end{array}\right)=\frac{1}{\sqrt{1+4 c}}\left[\left(\frac{1+\sqrt{1+4 c}}{2}\right)^{2 m+1}-\left(\frac{1-\sqrt{1+4 c}}{2}\right)^{2 m+1}\right]
$$

where $c \in \mathbb{C} \backslash\left\{0,-\frac{1}{4}\right\}$.

At the same time the formula (24) takes the form

$$
\sum_{k=0}^{m}\left(-\frac{1}{4}\right)^{k}\left(\begin{array}{c}
2 m-k \\
k
\end{array}\right)=\frac{2 m+1}{4^{m}}
$$

\section{Remark 2}

If $n$ is odd, $n=2 m+1$, then $\left\lfloor\frac{n}{2}\right\rfloor=\left\lfloor\frac{2 m+1}{2}\right\rfloor=\left\lfloor m+\frac{1}{2}\right\rfloor=m$ and the formula (22) has the form

$$
\sum_{k=0}^{m} c^{k}\left(\begin{array}{c}
2 m+1-k \\
k
\end{array}\right)=\frac{1}{\sqrt{1+4 c}}\left[\left(\frac{1+\sqrt{1+4 c}}{2}\right)^{2 m+2}-\left(\frac{1-\sqrt{1+4 c}}{2}\right)^{2 m+2}\right],
$$

where $c \in \mathbb{C} \backslash\left\{0,-\frac{1}{4}\right\}$. 
Whilst the formula (24) takes the form

$$
\sum_{k=0}^{m}\left(-\frac{1}{4}\right)^{k}\left(\begin{array}{c}
2 m+1-k \\
k
\end{array}\right)=\frac{m+1}{4^{m}}
$$

\section{Remark 3}

Let us observe that when $\Delta$ is a negative real or complex number then application of the formula (22) requires using of the de Moivre formula. For example if $c=-1$ then we have

$$
\sum_{k=0}^{\left\lfloor\frac{n}{2}\right\rfloor}(-1)^{k}\left(\begin{array}{c}
n-k \\
k
\end{array}\right)=\frac{2 \sqrt{3}}{3}(-1)^{n} \sin \frac{2(n+1) \pi}{3}
$$

\section{Conclusions}

It was shown that the second-order homogeneous linear difference equation with constant coefficients can be used for the calculation of the determinant of tridiagonal matrix with the same elements on particular diagonals. It can be observed that if on particular diagonals we have different elements then the calculation of determinant leads to second-order homogeneous linear difference equations with variable coefficients. The exact solutions of these equations can be obtained only in some special cases. The analysis of these special cases will be studied in the forthcoming paper.

\section{References}

[1] Biernat G., Boryś J., Całusińska I., Surma I., The three-band matrices, Scientific Research of the Institute of Mathematics and Computer Science 2008, 2(7), 119-122.

[2] Biernat G., Siedlecka U., Finite difference method in fourier equation internal case - direct formulas, Scientific Research of the Institute of Mathematics and Computer Science 2011, 2(10), 11-16.

[3] Feng J., Fibonacci identities via the determinant of tridiagonal matrix, Applied Mathematics and Computation 2011, 217, 5978-5981.

[4] Nalli A., Civciv H., A generalization of tridiagonal matrix determinants, Fibonacci and Lukas numbers, Chaos, Solitons and Fractals 2009, 40, 355-361.

[5] El-Mikkawy M., A note on a three-term recurrence for a tridiagonal matrix, Applied Mathematics and Computation 2003, 139, 503-511.

[6] Kucharzewski M., Piwko J., Równania różniczkowe i różnicowe, Wydawnictwo Politechniki Śląskiej, Gliwice 1977 (in Polish). 